\title{
Trigger factor in complex with the ribosome forms a molecular cradle for nascent proteins
}

Lars Ferbitz ${ }^{1}$, Timm Maier ${ }^{1}$, Holger Patzelt ${ }^{2}$, Bernd Bukau ${ }^{2}$, Elke Deuerling ${ }^{2}$ \& Nenad Ban ${ }^{1}$

${ }^{1}$ Institut für Molekularbiologie und Biophysik, Eidgenössische Technische Hochschule Hönggerberg (ETH Zürich), HPK Gebäude, CH-8093 Zürich, Switzerland

${ }^{2}$ Zentrum für Molekulare Biologie (ZMBH), Universität Heidelberg, Im Neuenheimer Feld 282, D-69120 Heidelberg, Germany

Konstanzer Online-Publikations-System (KOPS) URL: http://www.ub.uni-konstanz.de/kops/volltexte/2007/3706/ URN: http://nbn-resolving.de/urn:nbn:de:bsz:352-opus-37064
During protein biosynthesis, nascent polypeptide chains that emerge from the ribosomal exit tunnel encounter ribosomeassociated chaperones, which assist their folding to the native state $^{1,2}$. Here we present a $2.7 \AA$ crystal structure of Escherichia coli trigger factor, the best-characterized chaperone of this type, together with the structure of its ribosome-binding 
domain in complex with the Haloarcula marismortui large ribosomal subunit. Trigger factor adopts a unique conformation resembling a crouching dragon with separated domains forming the amino-terminal ribosome-binding 'tail', the peptidylprolyl isomerase 'head', the carboxy-terminal 'arms' and connecting regions building up the 'back'. From its attachment point on the ribosome, trigger factor projects the extended domains over the exit of the ribosomal tunnel, creating a protected folding space where nascent polypeptides may be shielded from proteases and aggregation. This study sheds new light on our understanding of co-translational protein folding, and suggests an unexpected mechanism of action for ribosome-associated chaperones.

In all organisms, messenger RNA-directed protein synthesis is achieved by the ribosome. The atomic structures of the large (50S) and small (30S) ribosomal subunits and the structural study of the entire bacterial ribosome provided important insights into this sophisticated macromolecular machine $e^{3}$. The structure of the H. marismortui 50 S subunit revealed a tunnel of approximately $100 \AA$ in length and $15 \AA$ in diameter, through which the polypeptide extends while still connected to the peptidyl transferase centre $^{4,5}$. The tunnel is long enough to accommodate a 35 -aminoacid-long segment of a nascent polypeptide in an extended conformation $^{6}$. The diameter of the tunnel allows for a helical structure of the peptide, but it is unlikely that significant protein folding can occur beyond helix formation ${ }^{5}$.

The initial folding events inside the ribosomal tunnel, however, are only the first steps in the complex process of folding newly synthesized proteins to their native three-dimensional structure. Whereas some proteins were reported to fold productively in a posttranslational mode ${ }^{7,8}$, several studies demonstrated co-translational folding in the cytosol of prokaryotic and eukaryotic cells 9 . Sequential co-translational folding of domains could be particularly advantageous for the production of multi-domain proteins because it limits possible unproductive inter- and intramolecular interactions during the early folding steps.

The cellular strategy to promote both co- and post-translational folding involves a large arsenal of molecular chaperones ${ }^{1,2}$. These proteins are found in all kingdoms and can be divided into two groups. The first, mainly chaperones from the Hsp70/40- and Hsp60/10 families, bind to newly synthesized proteins as soluble components of the cytosol. The second group welcomes nascent chains on the ribosome by binding to both the ribosome and the nascent chain, thereby assisting protein folding during ongoing synthesis. The existence of ribosome-associated chaperones is a highly conserved principle in eukaryotes and prokaryotes, although the involved components differ between species. The mechanism of how these ribosome-associated chaperones support the folding of nascent polypeptides is still unclear.

The bacterial ribosome-associated chaperone trigger factor binds to nascent chains and associates with ribosomes in a 1:1 stoichiometry through interactions of its $\mathrm{N}$-terminal domain with the ribosomal protein $\mathrm{L} 23$, which is located next to the peptide tunnel exit $^{10}$. This association is crucial for its interaction with nascent polypeptides and its in vivo function ${ }^{10}$. Although trigger factor is not essential in E. coli, the combined absence of trigger factor and the cytosolic DnaK chaperone causes cell death above $30^{\circ} \mathrm{C}$ and is accompanied by the massive aggregation of more than 340 different newly synthesized proteins $s^{11,12}$. Trigger factor was biochemically defined as a three-domain protein composed of an N-terminal ribosome-binding domain, a central peptidyl-prolyl cis/trans isomerase (PPIase) domain and a large C-terminal portion of unknown function ${ }^{13}$. The atomic structures of the isolated $\mathrm{N}$-terminal and PPIase domains have been solved, however, without providing mechanistic insights into the action of trigger factor in protein folding ${ }^{14,15}$.

Here, we report the crystal structures of E. coli trigger factor and the N-terminal trigger factor fragment bound to $H$. marismortui 50S. Together, these results outline the structure of the trigger factor-ribosome complex and the molecular environment for the folding of newly synthesized polypeptides, and provide a structural basis for the understanding of this fundamental cellular process.

Full-length trigger factor crystallizes in space group $P 2_{1}$ with two molecules in the asymmetric unit. Phases were calculated from a multiple anomalous dispersion experiment on selenomethioninesubstituted trigger factor with data extending to $2.7 \AA$. These data were also used for structure refinement (Supplementary Table 1a). The conformation of both molecules in the asymmetric unit is identical apart from local flexibility in loop regions (Supplementary Fig. S2). Trigger factor folds into a unique shape resembling a crouching dragon with overall dimensions of $122 \times 59 \times 63 \AA^{3}$, much larger than expected for this $48-\mathrm{kDa}$ protein (Fig. 1a).

The N-terminal domain of trigger factor ('tail') is exclusively responsible for the interactions with the non-translating ribosome ${ }^{10,13}$. Curiously, the second domain in sequence harbouring the PPIase activity is located on the opposite side of the molecule ('head'). It is connected to the N-terminal domain by means of a long linker extending along the 'back' of trigger factor. The $\mathrm{C}$-terminal domain is mostly $\alpha$-helical. It contributes to the formation of the 'back' of trigger factor and adds two extended 'arms' in the core of the protein. This domain is structurally similar to the chaperone domain of SurA, a protein that participates in the folding of outer membrane proteins in Gram-negative bacteria, although there is no sequence homology ${ }^{16}$. A large cradle is formed between the N-terminal ribosome-binding 'tail' and the C-terminal 'arms' of trigger factor. The analysis of crystal packing reveals the general peptide binding capacity of this 'chaperone' region. Trigger factor forms an extensive network of intermolecular contacts burying $3,500 \AA^{2}$ of surface area through intertwining of the 'arms' and the inner portion of the ribosome-binding domain. In one of the observed interactions, a neighbouring molecule inserts an $\alpha$-helix and a $\beta$-strand between the 'arms' of the $\mathrm{C}$-terminal domain, which is the narrowest part of the cradle (Supplementary Fig. S3). Even larger structural features could be accommodated between the ribosomebinding domain and the C-terminal 'arms'.

To understand the function of trigger factor as a ribosomeassociated chaperone, we co-crystallized the ribosome-binding domain of trigger factor and the large ribosomal subunit (50S). Owing to the suitability of the $H$. marismortui $50 S$ for crystallization we have chosen to form a heterologous complex between E. coli trigger factor and $H$. marismortui $50 \mathrm{~S}$. We could show conservation of the binding site between the bacterial chaperone and the archaeal ribosome by demonstrating that trigger factor binds with a 1:1 stoichiometry and can be crosslinked to ribosomal protein L23, in an identical way to that observed for the homologous complex (Supplementary Fig. S4). H. marismortui 50S was co-crystallized with the 144-amino-acid $\mathrm{N}$-terminal ribosome-binding fragment of trigger factor yielding crystals that are isomorphous to crystals of isolated 50S. This strategy was best suited for obtaining structural data on the trigger factor-ribosome complex because full-length trigger factor interferes with the $50 \mathrm{~S}$ crystal packing, whereas its connection to the ribosome is too delicate to support alternative packing modes.

Data from co-crystals were collected to $3.5 \AA$ resolution (Supplementary Table $1 \mathrm{~b}$ ) and Fourier difference maps revealed electron density for the ribosome-binding region of trigger factor, which contains the 'signature motif' ${ }_{43} \mathrm{GFRxGxx}_{50}$ ) and two surrounding $\alpha$-helices (amino acids 20-39 and 50-59). From its anchor point on the ribosome, the trigger factor fragment is visible up to a distance of $20 \AA$ above the 50 S surface before the density fades out due to increasing temperature factors. Although flexible in the uncomplexed form, one of the previously observed conformations of the ribosome-binding loop (Protein Data Bank: 1OMS_B; ref. 14) 
can be unambiguously placed into the electron density using a kink in one helix and bulky residues as points of reference (Fig. 1b). Starting from the structure of 50S (Protein Data Bank:1JJ2) and the fitted fragment of trigger factor (amino acids 25-59), rigid body fitting, positional and temperature factor optimization and manual rebuilding in the attachment region yielded a model with an $R$-factor of $R / R_{\text {free }}=0.192 / 0.268$.

Trigger factor binds the ribosome at the triple junction between domain III of $23 \mathrm{~S}$ ribosomal RNA and proteins L23 and L29, proximal to the exit of the ribosomal nascent chain tunnel (Fig. 1c). Overall, $1,227 \AA^{2}$ of surface area is buried upon complex formation, which is divided into $423 \AA^{2}, 622 \AA^{2}$ and $182 \AA^{2}$ for the contact of trigger factor with $23 \mathrm{~S}$ rRNA, L23 and L29, respectively. Because the proteins at the interface form a ridge, only residues at the tip of the ribosome-binding loop around Arg 45 of trigger factor extend far enough to reach the rRNA. Hydrophobic interactions with L23 involve three residues of trigger factor (Phe 44, Pro 50 and Ile 53) surrounding Met 16 on L23 (Fig. 1d). This residue is replaced by phenylalanine in the E. coli L23 protein, which would result in even tighter hydrophobic interactions. The most important interaction is mediated by Glu 13 on L23, which was shown to be essential for trigger factor binding to $50 \mathrm{~S}^{10}$. This residue has an altered side-chain conformation in the complex structure and, with two hydrogen bonds, positions the side chain of Arg 45 of trigger factor for hydrophobic stacking with the unpaired RNA base, and for salt interactions with the backbone phosphate of A1501 in 50S (E. coli: A1392) (Fig. 1d). The binding region of L23 and the secondary structure and sequence of rRNA around A1501 are highly conserved in archaea and bacteria (Supplementary Fig. S5a, b). In a second and minor interaction site the C-terminal residues Val 84 and Phe 85 of L23, which were not visible in the isolated 50S crystal structure, form weak hydrophobic contacts with Ile 53 and
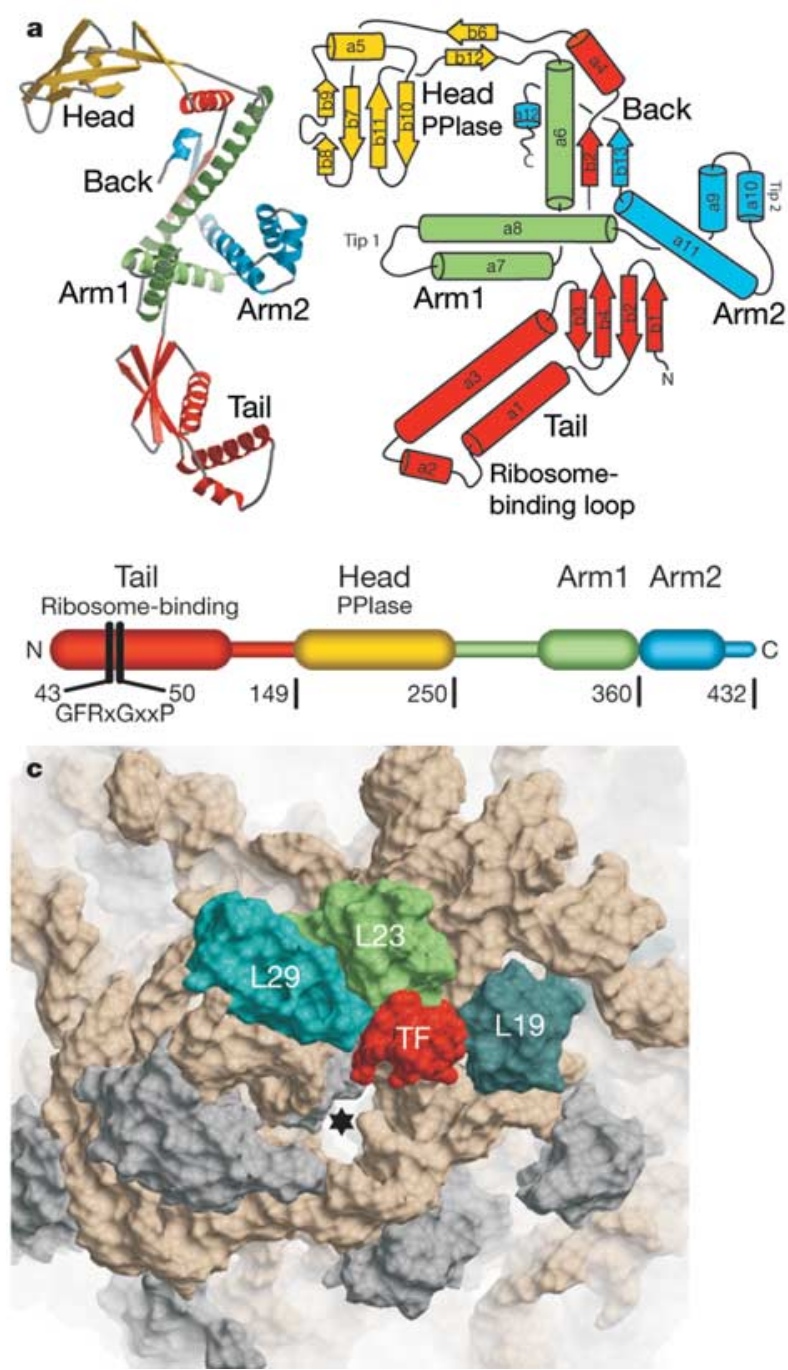

Figure 1 Structure of $E$. coli trigger factor and its $\mathrm{N}$-terminal domain bound to the $50 \mathrm{~S}$ ribosomal subunit. a, Trigger factor adopts an extended fold. Left: ribbon diagram of the trigger factor fold. Right: schematic representation of the domain organization. Bottom: domain arrangement in sequence space. Positions of the ribosome-binding trigger factor signature (residues 43-50) and domain borders are indicated. In all parts the ribosome binding 'tail' is shown in red, the PPlase 'head' in yellow and 'arm' 1 and 'arm' 2 in green and blue, respectively. b. Trigger factor fragment 1-144 bound to the 50S ribosomal subunit: simulated annealed omit map around residues 26-59 of the bound trigger factor 1-144 fragment shown together with a surface representation of 50S; key interacting
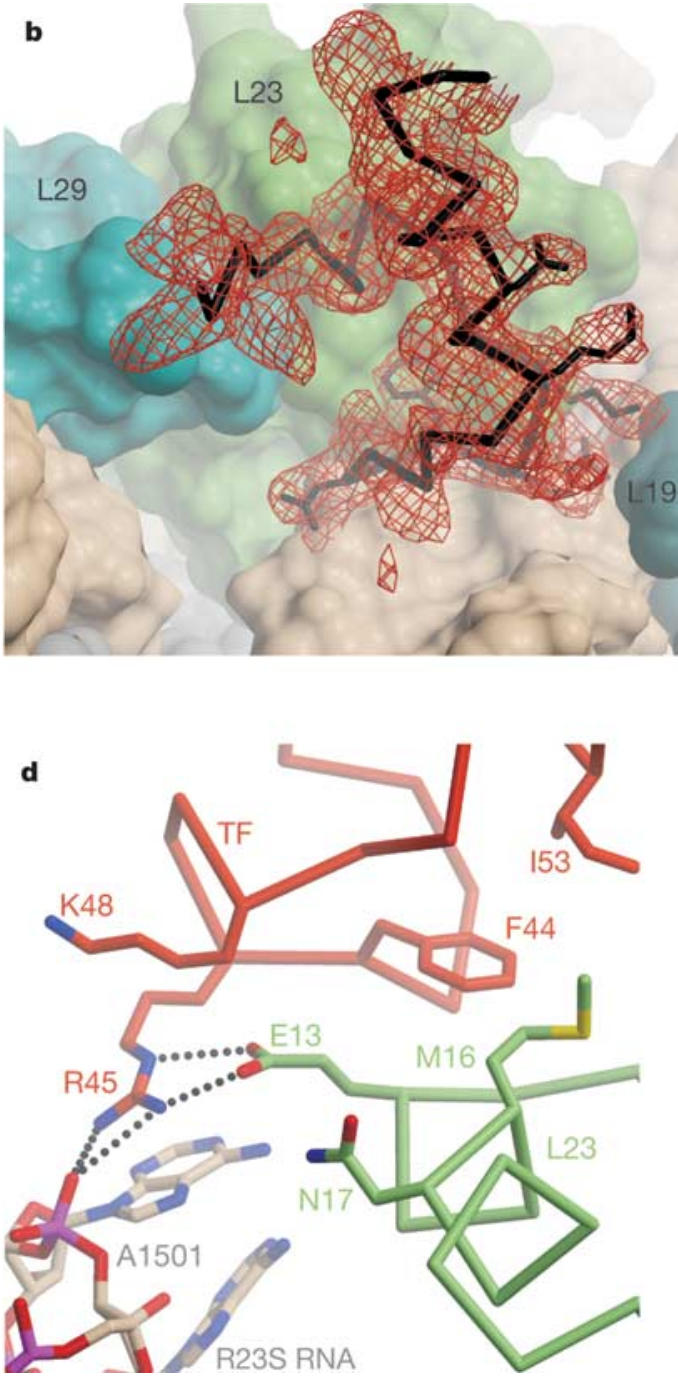

proteins are labelled. For clarity, only selected side chains are shown. c, Same site as in b viewed from a distance; trigger factor fragment is shown as a red surface representation and the peptide exit tunnel is denoted with an asterisk. In $\mathbf{b}$ and $\mathbf{c}$ rRNA is coloured biege; non-interacting proteins, grey; L29, turquoise; L23, green; and L19, bluish-green. d, Key interactions between the trigger factor (TF) fragment (red), L23 (green) and 23S rRNA. Selected residues are shown together with $\mathrm{C}_{\alpha}$-traces. Hydrogen bonds are indicated only for the interaction between the key contact residues E13 (L23), R45 (trigger factor) and A1501 (R23S). 
Asn 52 of trigger factor, respectively. Contacts with ribosomal proteins L29 and L19 are tangential and are unlikely to contribute to the trigger factor binding affinity, in agreement with biochemical data $^{10}$.

The structure of full-length trigger factor, together with that of its ribosome-binding fragment in complex with 50S, permits accurate placement of the entire chaperone on the ribosome through superposition of the ribosome-binding region that is visible in both structures. Judging from the increasing temperature factors of the helices in the co-crystallized fragment, full-length trigger factor bound to the ribosome could swing, on average, by $10^{\circ}$ in all directions around its attachment point. On the basis of the superposition of the two full-length trigger factor molecules in the crystal (Supplementary Fig. S2) and the presence of a well-defined electron density for the interdomain connections, we have no indications for potential domain rearrangements. The peripheral mode of interaction of trigger factor with $50 \mathrm{~S}$ is also unlikely to induce any remote domain motions in trigger factor. However, the presence of additional conformational states of ribosome-bound trigger factor cannot be ruled out completely.

Trigger factor hunches over the polypeptide exit of the ribosome and extends its sticky, hydrophobic inner face towards the area of the nascent polypeptide exit (Fig. 2a-c). This evokes a model in which nascent chains initially interact with the cradle of the trigger factor surrounded by helices belonging to the ribosome-binding domain and the 'arms' of the chaperone. Although trigger factor hunches over the exit and provides a significantly shielded environment for the newly synthesized peptides, no region of the chaperone other than the 'tail' contacts the ribosome (Fig. 2a). The closest approach from the ribosomal surface to the tips of the C-terminal 'arms' is around $10 \AA$ whereas the distance to the bottom of the cradle is $40 \AA$ (Fig. 3b). The charge distribution of trigger factor shows an accumulation of basic residues at the ribosome-binding 'tail' and of hydrophobic residues both in parts of the N-terminal domain and the C-terminal 'arms', supposed to be the contact regions of the cradle for emerging nascent polypeptides (Fig. 3a). These findings agree with biochemical data that demonstrate that trigger factor lacking the PPIase domain still binds peptides enriched in hydrophobic residues ${ }^{17}$.

Although the role of trigger factor in protein folding is firmly established, it has been difficult to study its detailed mechanism of action. The role of its C-terminal domain was particularly puzzling because, as an isolated fragment, it is structurally unstable and without assignable function ${ }^{13,17}$. The atomic structure of trigger factor now provides an explanation for these difficulties. The 'back' of the C-terminal domain is critically stabilized by residues 111133, which connect the N-terminal and the PPIase domain, and therefore may not preserve its conformation in the absence of this connection. Furthermore, from the structure it is not surprising that this domain shows little sequence conservation. It is likely that only the overall shape and the surface distribution of hydrophobicity are critical for substrate binding, and that, due to the diverse nature of nascent peptides, there is little selective pressure to maintain exact positioning of particular side chains.

The C-terminal and the N-terminal domain together form a cradle whose inner surface appears to be tailored to interact with unfolded proteins. Moreover, this cradle is optimally positioned at the exit site of the ribosomal tunnel for the capture of nascent

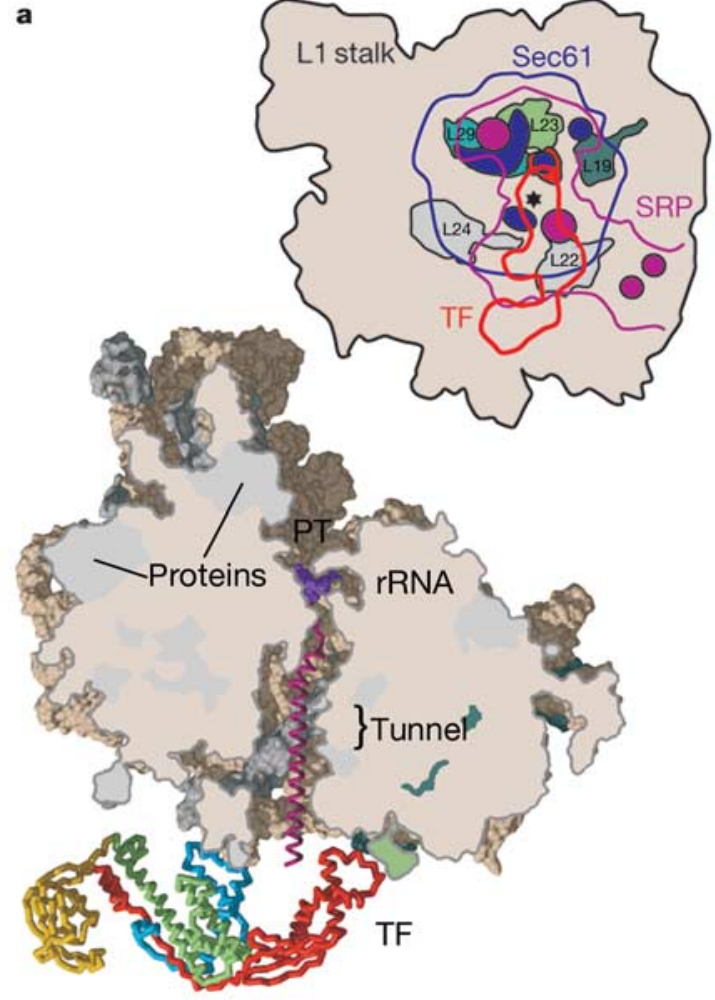

Figure 2 Structure of the trigger factor bound to the 50 S ribosomal subunit. a, Overview of the trigger factor $50 \mathrm{~S}$ complex. Full-length trigger factor positioned by superimposition onto the ribosome-bound fragment trigger factor $1-144$ is shown as $\mathrm{C}_{\alpha}$-trace together with a slice of $50 \mathrm{~S}$ along the peptide exit tunnel (for clarity, further cavities peripheral to the tunnel are not shown) with a modelled nascent chain in magenta, extending from the peptidyl transferase centre (PT). Colouring is as in Fig. 1. Inset: schematic footprints of
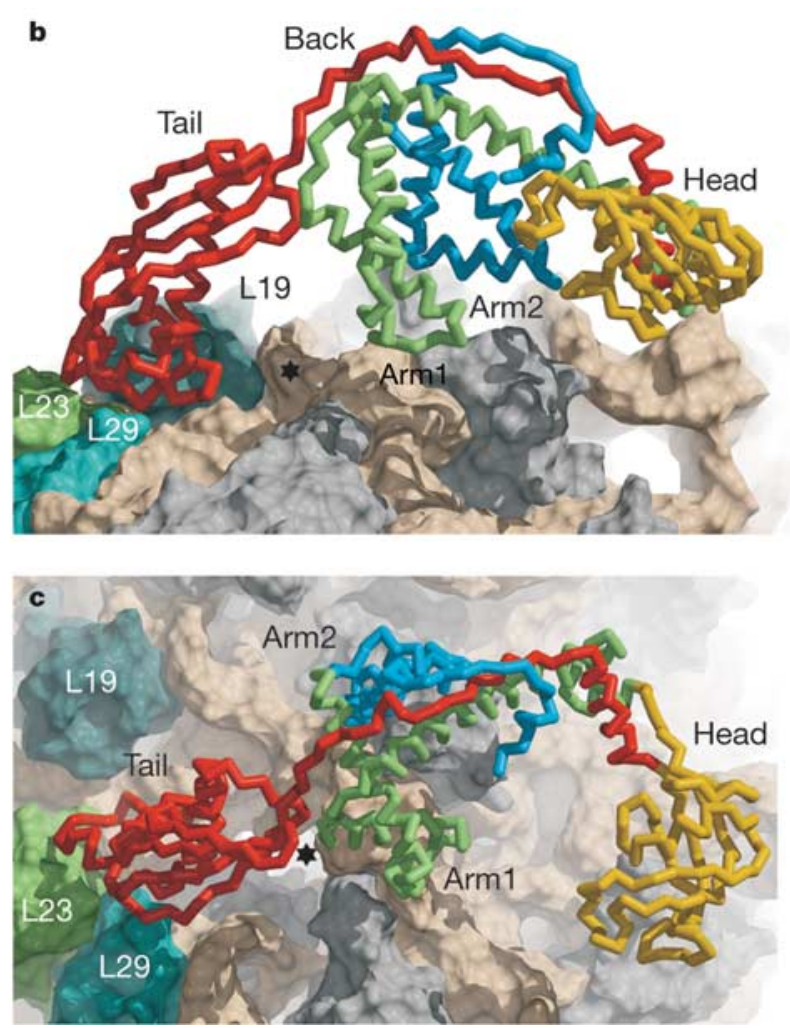

Sec61, SRP and the trigger factor on the ribosome on the basis of crystallographic and electron microscopic data. Binding sites for Sec61p (blue), SRP (magenta) and the trigger factor (red) are represented as filled areas, and projections of the molecules are shown as outlines in the same colours. Positions of selected ribosomal proteins are indicated in the same colours as in Fig. 1. b, c, Close-up side (b) and top (c) views of the complex shown in a without a nascent peptide. 


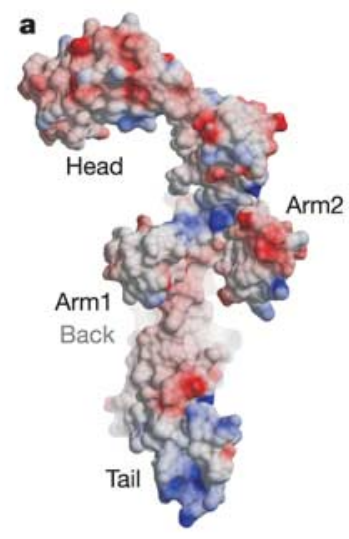

Figure 3 Trigger factor exposes a hydrophobic cradle to the nascent chain. a, Solvent accessible surface of trigger factor (in stereo view), coloured by electrostatic potential

polypeptides. Its role as the major substrate-binding site of trigger factor is further indicated by several pieces of evidence: (1) there is significant hydrophobicity over the entire inner surface of the cradle (Fig. 3a); (2) the fused N-terminal and C-terminal domains provide trigger factor with almost wild-type-like chaperone activity in vivo and in vitro ${ }^{17}$ as well as peptide binding activity, even in the absence of the PPIase domain (see below); (3) the cradle is involved in forming an extensive network of crystallographic intermolecular contacts as described above; and (4) the structurally similar domain of the SurA chaperone has substrate-binding properties $^{19}$.

Recently, it has been shown that PPIase activity is not essential for nascent chain binding and chaperone activity of trigger factor ${ }^{18}$. In agreement with these findings, the PPIase domain is peripheral to the main substrate-binding cradle of trigger factor and to the exit of the ribosomal tunnel and is tethered to the body of trigger factor by means of a double linker. As a consequence, the PPIase activity will be limited to regions above the 'arms' and behind the 'back' of trigger factor where partially folded proteins will appear only after they have escaped from the trigger factor cradle. The PPIase domain will therefore interact with nascent chains at later stages of their synthesis, restricting its catalytic activity to selected sites that remain accessible at this folding stage.

Dimerization of trigger factor in solution has been observed in vitro, raising possible implications for the mechanism of its action $^{17,20}$. Crystallographic data presented here show no evidence of a perfect dimer formation. Trigger factor molecules in the crystal form a network of contacts mainly through residues implicated in formation of the substrate-binding cradle and thus may mimic the interactions between trigger factor and its nascent chain substrates as discussed before (Supplementary Fig. S3). These interactions are not possible when trigger factor binds the ribosome because the hydrophobic contact surface orients towards the ribosomal exit tunnel, consistent with the observation that it binds the ribosome as a monomer ${ }^{20,21}$. Dimerization as observed in solution may help to prevent aggregation and unwanted substrate interactions of trigger factor in the cytosol ${ }^{20}$.

Trigger factor has inherent affinity for ribosomes, which is increased when they display nascent peptides ${ }^{21}$. The weak affinity of trigger factor to the ribosome $\left(K_{\mathrm{d}}=1.2 \mu \mathrm{M}^{20,22}\right)$ and its small anchor point may be ideally suited for its role in protein folding. By maintaining a precise balance of binding affinities, trigger factor dissociates from vacant ribosomes within seconds ${ }^{22}$, but may become stabilized by hydrophobic interactions with an emerging, unfolded nascent peptide. The broad distribution of hydrophobicity within the trigger factor cradle may allow extensive but transient contacts of trigger factor with the emerging chain,

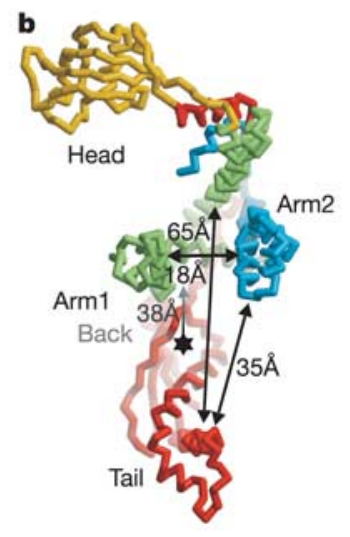

(blue, positive; red, negative). $\mathbf{b}, \mathrm{C}_{\alpha}$-trace representation of trigger factor with approximate dimensions of the cradle indicated; coloured according to Fig. 1.

consistent with the observation that the association of trigger factor with the translating ribosomes becomes salt insensitive $e^{1,21}$. Once a domain folds co-translationally, it buries hydrophobic residues, detaches from trigger factor and destabilizes the inter-

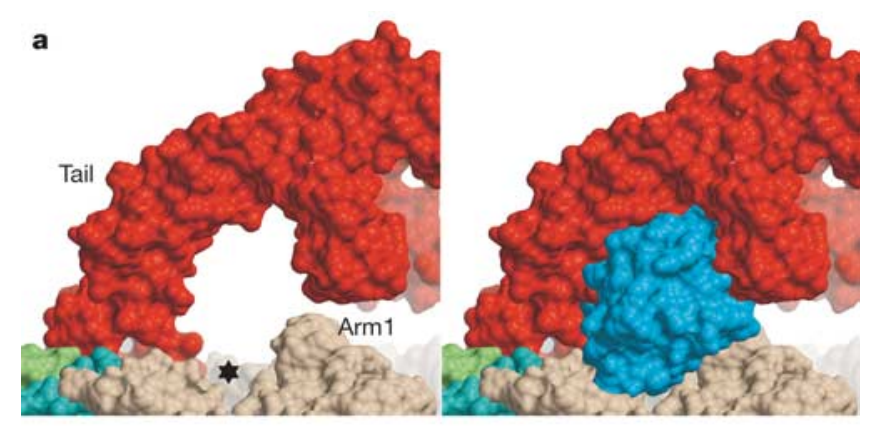

b
1

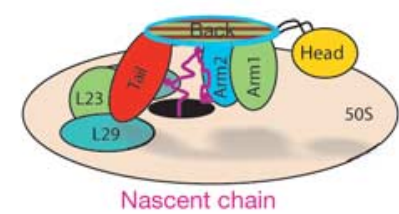

3

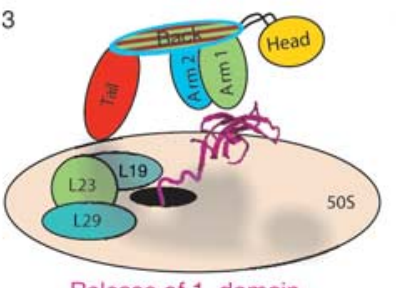

Release of 1. domain
2
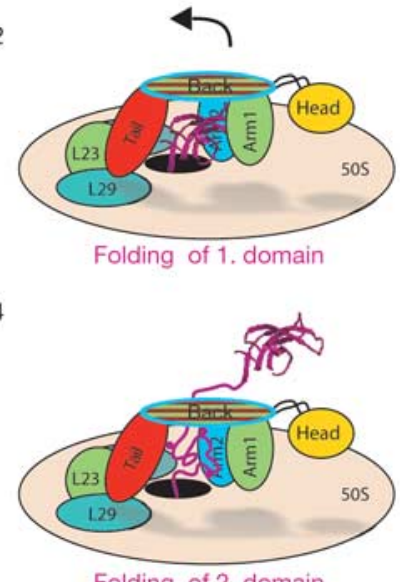

Figure $4 \mathrm{~A}$ model for the interaction of trigger factor with nascent chains. a, The trigger factor cradle could accommodate entire protein domains. Left: view through the arch-like structure delineated by the tail and arms of trigger factor (red) covering the peptide exit tunnel, indicated by an asterisk. Right: identical view to the lefthand figure; the size of the arch can accommodate an entire molecule of lysozyme (blue), manually fitted into this region for illustration. $\mathbf{b}$, Schematic representation of the proposed mechanism of trigger factor action and its possible role in facilitating domain-wise co-translational protein folding. Colours are as in Fig. 2. Initially (1) the trigger factor is bound to an unfolded nascent chain. Upon folding of this domain (2), contacts between the trigger factor and the newly synthesized peptide are weakened and trigger factor dissociates from the ribosome (3). The trigger factor re-associates with the ribosome when the next stretch of newly synthesized, unfolded polypeptide becomes exposed (4). 
action of trigger factor with the ribosome-nascent chain complex (Fig. 4b).

We conclude that trigger factor might promote co-translational folding of domains by providing a shielded environment in which the folding is initially postponed through hydrophobic contacts, but then allowed to proceed, perhaps in a self-promoted fashion, when sufficient sequence information is available for the generation of a folded core (Fig. 4b). The chaperone would then dissociate from the ribosome and rebind once a significant new portion of the unfolded polypeptide becomes exposed at the tunnel exit. The folding space formed between trigger factor and the ribosome is sufficiently large to accommodate a domain of the size of the $14-\mathrm{kDa}$ protein lysozyme (Fig. 4a). A different option, which we consider less likely on the basis of available biochemical evidence, is that trigger factor remains bound during the synthesis of the entire polypeptide, even when multi-domain proteins are translated. In this case, the co-translationally folded domain could escape through the relatively large gaps between the ribosome and the chaperone arch on either side.

Trigger factor is just one of the macromolecular factors that bind to ribosome-nascent chain complexes. The signal recognition particle (SRP) targets ribosomes displaying signal sequences to membranes where the newly synthesized proteins are co-translationally translocated ${ }^{23}$. At the membranes, the ribosome-nascent chain complexes are bound to the translocon, a protein-conducting channel. Both SRP and the translocon have now been visualized on the eukaryotic ribosome by electron microscopy ${ }^{24,25}$. Because protein folding and targeting require precise exchange between all these factors it is interesting to compare their binding sites on the ribosome. Both SRP and the eukaryotic protein-conducting channel, Sec61, use eukaryotic homologues of ribosomal proteins L23 and L29 as major attachment sites, analogous to what is reported here for trigger factor. These ribosomal proteins have also been crosslinked to the protein component of SRP (SRP54) ${ }^{26}$. It is tempting to speculate that all factors transiently associated with ribosome-nascent chain complexes will use a similar mode of interaction characterized by low affinity, loop-mediated binding to the ribosome augmented by contacts to the nascent chain. Interestingly, one of the attachment sites for Sec61 coincides with those of trigger factor (Fig. 2a).

Here we have shown that trigger factor, together with the ribosome, forms a well-defined protective cage of sufficient size to accommodate a folded protein domain at the peptide tunnel exit site. This is in contrast to the previous assumption that the $48-\mathrm{kDa}$ trigger factor can only bind small stretches of unfolded peptides but not provide a shielded folding space. The two modes of action may not be mutually exclusive and may depend on the nature of the nascent polypeptide. The structural information presented here will serve as a basis for functional experiments aimed at addressing the mechanism of protein folding facilitated by ribosome-associated chaperones.

\section{Methods}

\section{Crystallization of trigger factor and trigger factor-50S complexes}

Full-length trigger factor and its N-terminal fragment consisting of the first 144 amino acids were expressed and purified as previously described ${ }^{18}$. We obtained crystals of the full-length trigger factor in $22-26 \%(\mathrm{w} / \mathrm{v})$ polyethylene glycol 4,000 with $14 \%(\mathrm{v} / \mathrm{v})$ 1,4-butanediol in 0.1 M MES (2-N-morpholino-ethanesulphonic acid) at pH 7.0-7.5. Crystals grew to a maximum size of $0.2 \times 0.3 \times 1.0 \mathrm{~mm}^{3}$ in four to six weeks at $4{ }^{\circ} \mathrm{C}$ and belong to space group $P 2_{1}\left(\mathrm{a}=100.2 \AA, \mathrm{b}=47.4 \AA, \mathrm{c}=114.8 \AA, \beta=113.6^{\circ}\right)$ with two molecules in the asymmetric unit. Selenomethionine-labelled protein was produced with standard procedures and crystallized under similar conditions. The N-terminal fragment of trigger factor was used at tenfold molar excess over $50 \mathrm{~S}$ for co-crystallization experiments. Crystals were obtained under conditions previously described for $50 \mathrm{~S}$ crystallization ${ }^{4}$ and belong to space group $C 222_{1}(a=211 \AA, b=299 \AA, c=574 \AA)$ with one molecule in the asymmetric unit.

\section{Data collection}

Full-length trigger factor crystals were stabilized by stepwise addition of 2-methyl-2,4pentane diol (MPD) to yield a final concentration of $25 \%(\mathrm{v} / \mathrm{v})$. Crystals were flash frozen in liquid propane. All measurements were done at the Swiss Light Source beamline X06SA at the Paul Scherrer Institute at $100 \mathrm{~K}$. A native data set to $2.8 \AA$ resolution and a selenomethionine derivative data set at inflection point, peak and remote wavelength to $2.7 \AA$ were collected. The latter was used for multiwavelength anomalous dispersion phasing (Supplementary Table 1a) and, owing to the large non-isomorphism between crystals, also for refinement.

Crystals of $50 \mathrm{~S}$ in complex with the ribosome-binding domain of trigger factor were stabilized as described and flash frozen in liquid propane ${ }^{4}$. Complete data sets were collected at the Swiss Norwegian Beamline at ESRF and the Swiss Light Source to $3.5 \AA$ resolution (Supplementary Table $1 \mathrm{~b}$ ). All data were integrated and scaled using $\mathrm{HKL}^{27}$.

\section{Structure determination}

All phasing and refinement was done using the program $\mathrm{CNS}^{28}$. For the full-length trigger factor data, an initial set of heavy atom sites was identified in the anomalous difference Patterson maps by automatic correlation coefficient search procedures. Additional sites were found in difference Fourier maps after improving the phases by solvent flipping. In total, 19 selenium sites were used for phasing. Maximum likelihood refinement with amplitudes and experimental phase probability distribution was used during model building. The structure was refined to $2.7 \AA$ resolution with $R / R_{\text {free }}=0.241 / 0.324$ with an average B-value of $74 \AA^{2}$. Improved maps were calculated as prime-and-switch composite omit maps using the program RESOLVE ${ }^{29}$. The $\mathrm{N}$-terminal ribosome binding domain of the trigger factor in complex with the $50 \mathrm{~S}$ was located in the $F_{\mathrm{o}}-F_{\mathrm{c}}$ difference Fourier maps. The structure of the complex was refined to $3.5 \AA$ resolution with $R / R_{\text {free }}=0.192$ / 0.268. All models were built using the program $\mathrm{O}^{30}$. For details of figure preparation see Supplementary Information.

\section{In vitro crosslinking}

$1 \mu \mathrm{M}$ purified $50 \mathrm{~S}$ ribosomal $H$. marismortui subunits were incubated with $2 \mu \mathrm{M}$ mutant trigger factor V49C coupled to the UV-inducible crosslinker BPIA (benzophenone-4iodoacetamide, Molecular Probes, Inc.) for $30 \mathrm{~min}$ at $30^{\circ} \mathrm{C}$ in the presence of $0.6 \mathrm{M} \mathrm{NaCl}$ as described ${ }^{10}$. Samples were irradiated with UV light on ice for $5 \mathrm{~min}(365 \mathrm{~nm}, 100 \mathrm{~W}$; Ultraviolet Products, Model B-100AP) at a distance of $5 \mathrm{~cm}$. Ribosomal complexes were separated by centrifugation through sucrose cushions ${ }^{13}$. Protein identification was done by in-gel-digest of the crosslinking products with trypsin followed by mass spectrometry ${ }^{10}$

Received 25 May; accepted 27 July 2004; doi:10.1038/nature02899.

Published online 29 August 2004

1. Bukau, B., Deuerling, E., Pfund, C. \& Craig, E. A. Getting newly synthesized proteins into shape. Cell 101, 119-122 (2000).

2. Hartl, F. U. \& Hayer-Hartl, M. Molecular chaperones in the cytosol: from nascent chain to folded protein. Science 295, 1852-1858 (2002).

3. Ramakrishnan, V. Ribosome structure and the mechanism of translation. Cell 108, 557-572 (2002).

4. Ban, N., Nissen, P., Hansen, J., Moore, P. B. \& Steitz, T. A. The complete atomic structure of the large ribosomal subunit at $2.4 \AA$ resolution. Science 289, 905-920 (2000).

5. Nissen, P., Hansen, J., Ban, N., Moore, P. B. \& Steitz, T. A. The structural basis of ribosome activity in peptide bond synthesis. Science 289, 920-930 (2000).

6. Hardesty, B. \& Kramer, G. Folding of a nascent peptide on the ribosome. Prog. Nucleic Acid Res. Mol. Biol. 66, 41-66 (2001).

7. Agashe, V. R. et al. Function of trigger factor and $\mathrm{DnaK}$ in multidomain protein folding: increase in yield at the expense of folding speed. Cell 117, 199-209 (2004).

8. Netzer, W. J. \& Hartl, F. U. Recombination of protein domains facilitated by co-translational folding in eukaryotes. Nature 388, 343-349 (1997).

9. Nicola, A. V., Chen, W. \& Helenius, A. Co-translational folding of an alphavirus capsid protein in the cytosol of living cells. Nature Cell Biol. 1, 341-345 (1999).

10. Kramer, G. et al. L23 protein functions as a chaperone docking site on the ribosome. Nature 419 , 171-174 (2002).

11. Deuerling, E., Schulze-Specking, A., Tomoyasu, T., Mogk, A. \& Bukau, B. Trigger factor and DnaK cooperate in folding of newly synthesized proteins. Nature 400, 693-696 (1999).

12. Teter, S. A. et al. Polypeptide flux through bacterial Hsp70: DnaK cooperates with Trigger Factor in chaperoning nascent chains. Cell 97, 755-765 (1999).

13. Hesterkamp, T., Deuerling, E. \& Bukau, B. The amino-terminal 118 amino acids of Escherichia coli trigger factor constitute a domain that is necessary and sufficient for binding to ribosomes. J. Biol. Chem. 272, 21865-21871 (1997).

14. Kristensen, O. \& Gajhede, M. Chaperone binding at the ribosomal exit tunnel. Structure (Camb.) 11, $1547-1556$ (2003).

15. Vogtherr, M. et al. NMR solution structure and dynamics of the peptidyl-prolyl cis-trans isomerase domain of the trigger factor from Mycoplasma genitalium compared to FK506-binding protein. J. Mol. Biol. 318, 1097-1115 (2002)

16. Bitto, E. \& McKay, D. B. The periplasmic molecular chaperone protein SurA binds a peptide motif that is characteristic of integral outer membrane proteins. J. Biol. Chem. 278, 49316-49322 (2003).

17. Kramer, G. et al. Functional dissection of Escherichia coli trigger factor: unraveling the function of individual domains. J. Bacteriol. 186, 3777-3784 (2004).

18. Kramer, G. et al. Trigger factor peptidyl-prolyl cis/trans isomerase activity is not essential for the folding of cytosolic proteins in Escherichia coli. J. Biol. Chem. 279, 14165-14170 (2004).

19. Behrens, S., Maier, R., de Cock, H., Schmid, F. X. \& Gross, C. A. The SurA periplasmic PPIase lacking its parvulin domains functions in vivo and has chaperone activity. EMBO J. 20, 285-294 (2001).

20. Patzelt, H. et al. Three-state equilibrium of Escherichia coli trigger factor. Biol. Chem. 383, 1611-1619 (2002).

21. Hesterkamp, T., Hauser, S., Lütcke, H. \& Bukau, B. Escherichia coli trigger factor is a prolyl isomerase that associates with nascent polypeptide chains. Proc. Natl Acad. Sci. USA 93, 4437-4441 (1996)

22. Maier, R., Eckert, B., Scholz, C., Lilie, H. \& Schmid, F. X. Interaction of trigger factor with the ribosome. J. Mol. Biol. 326, 585-592 (2003). 
23. Schatz, G. \& Dobberstein, B. Common principles of protein translocation across membranes. Science 271, 1519-1526 (1996)

24. Beckmann, R. et al. Alignment of conduits for the nascent polypeptide chain in the ribosome-Sec6 complex. Science 278, 2123-2126 (1997).

25. Halic, M. et al. Structure of the signal recognition particle interacting with the elongation-arrested ribosome. Nature 427, 808-814 (2004)

26. Pool, M. R., Stumm, J., Fulga, T. A., Sinning, I. \& Dobberstein, B. Distinct modes of signal recognition particle interaction with the ribosome. Science 297, 1345-1348 (2002).

27. Otwinowski, Z. M. W. in Macromolecular Crystallography Part A Vol. 276 (ed. Carter, C. W. Jr, S.R.M.) 307-326 (Academic, San Diego, 1997).

28. Brunger, A. T. et al. Crystallography \& NMR system: A new software suite for macromolecular structure determination. Acta Crystallogr. D Biol. Crystallogr. 54, 905-921 (1998).

29. Terwilliger, T. C. \& Berendzen, J. Automated MAD and MIR structure solution. Acta Crystallogr. D Biol. Crystallogr. 55, 849-861 (1999).

30. Jones, T. A., Bergdoll, M. \& Kjeldgaard, O. in Crystallographic and Modeling Methods in Molecular Design (eds Bugg, C. \& Ealick, S.) 189-195 (Springer-Verlag, New York, 1990).

Supplementary Information accompanies the paper on www.nature.com/nature.
Acknowledgements Data collection was performed at the Swiss Light Source, Paul Scherrer Institut, Villigen and at the Swiss Norwegian Beamline (ESRF, Grenoble). We are grateful to C. Schulze-Briese, T. Tomizaki and A. Wagner at the SLS as well as P. Pattison and S. Capelli at the SNBL whose outstanding efforts have made these experiments possible. We also thank our colleagues S. Antolic, M. Steiner and members of the Ban laboratory for help in ribosome preparations and suggestions; and members of the Bukau laboratory for discussions. This work was supported by the Swiss National Science Foundation (SNSF), the NCCR Structural Biology program of the SNSF, an ETH research grant, grants of the Deutsche Forschungsgemeinschaft to B.B. and E.D. and a Young Investigator grant from the Human Frontier Science Program to N.B. and E.D.

Competing interests statement The authors declare that they have no competing financial interests.

Correspondence and requests for materials should be addressed to N.B. (ban@mol.biol.ethz.ch) or E.D. (e.deuerling@zmbh.uni-heidelberg.de).Structure coordinates have been deposited in the Protein Data Bank under the accession codes 1w26 and 1w2b. 